\title{
Recombinant human papillomavirus nonavalent vaccine in the prevention of cancers caused by human papillomavirus
}

This article was published in the following Dove Press journal:

Infection and Drug Resistance

Zheng Quan Toh ${ }^{1,2}$
Jennie Kosasih'

Fiona M Russell ${ }^{1,3}$

Suzanne M Garland ${ }^{4,5}$

Edward K Mulholland 1,6

Paul V Licciardi ${ }^{1,2}$

'Infection and Immunity, Murdoch Children's Research Institute, Parkville, Victoria, Australia; ${ }^{2}$ Department of Paediatrics, The University of Melbourne, Parkville, Victoria, Australia; ${ }^{3}$ Centre for International Child Health, Department of Paediatrics, The University of Melbourne, Parkville, Victoria, Australia; ${ }^{4}$ Department of Obstetrics and Gynaecology, The University of Melbourne, Parkville, Victoria, Australia; ${ }^{5}$ Regional WHO HPV Reference Laboratory, Centre for Women's Infectious Diseases Research, The Royal Women's Hospital, Parkville, Victoria, Australia; ${ }^{6}$ Department of Infectious Disease Epidemiology, London School of Hygiene and Tropical Medicine, University of London, London WCIE7HT, UK
Correspondence: Paul V Licciardi Murdoch Children's Research Institute, Royal Children's Hospital, Flemington Road, Parkville, Victoria 3052, Australia Tel +6 I39 3455554

Email paul.licciardi@mcri.edu.au

\begin{abstract}
Human papillomavirus (HPV) types 16 and 18 cause $70 \%$ of cervical cancer cases globally. The nonavalent HPV vaccine (9vHPV) was licensed in 2014 and protects against the next five most common cancer-causing HPV types (HPV 31/33/45/52/58) after HPV 16/18. Phase III clinical studies have demonstrated high vaccine efficacy $(>90 \%)$ against cervical, vulvar, and vaginal precancers caused by these additional types, and have shown comparable immunogenicity to the shared genotypes to quadrivalent HPV vaccine $(4 \mathrm{vHPV})$. Vaccine efficacy and antibody responses for 9vHPV are found to persist for at least five years while longer-term observational studies are ongoing to monitor long-term vaccine effectiveness. The implementation of 9vHPV has the potential to prevent up to $93 \%$ of cervical cancer cases, as well as a significant proportion of other HPV-related anogenital cancers. This review article summarizes the current evidence for 9vHPV in terms of vaccine efficacy against HPV infection and related anogenital precancers, safety, and immunogenicity, as well as discussing the potential impact of this vaccine on the cervical cancer burden globally.
\end{abstract}

Keywords: nonavalent human papillomavirus vaccine, review, efficacy, immunogenicity, safety

\section{Introduction}

The human papillomavirus (HPV) vaccines are made using recombinant technology based on the self-assembly nature of the L1 capsid protein of the virus (viral-like particles, VLPs). As the VLPs do not contain the viral genome, they are not infectious. There are currently three licensed prophylactic HPV vaccines against HPV infections and cervical cancer precursors. The timeline of the Phase III clinical trials and licensure/registration of HPV vaccines is shown in Figure 1, and countries that have a national HPV immunization program are shown in Figure 2. The firstgeneration HPV vaccines, quadrivalent (4vHPV) and bivalent (2vHPV), were first licensed in 2006 and 2007, respectively. Both vaccines protect against HPV types 16 and 18 (major types causing cervical cancers), while 4vHPV also protects against HPV types 6 and 11 (major types causing genital warts). In 2014, a secondgeneration nonavalent HPV vaccine (9vHPV) was licensed by The United States (U.S.) Food and Drug Administration (FDA) for individuals aged 9-26 years, and subsequently extended to women up to age 45 years in 2018. This vaccine contains an additional five cancer-causing HPV types (HPV31, 33, 45, 52, and 58) in addition to the four types in $4 \mathrm{vHPV}$, and has the potential to prevent up to $93 \%$ 


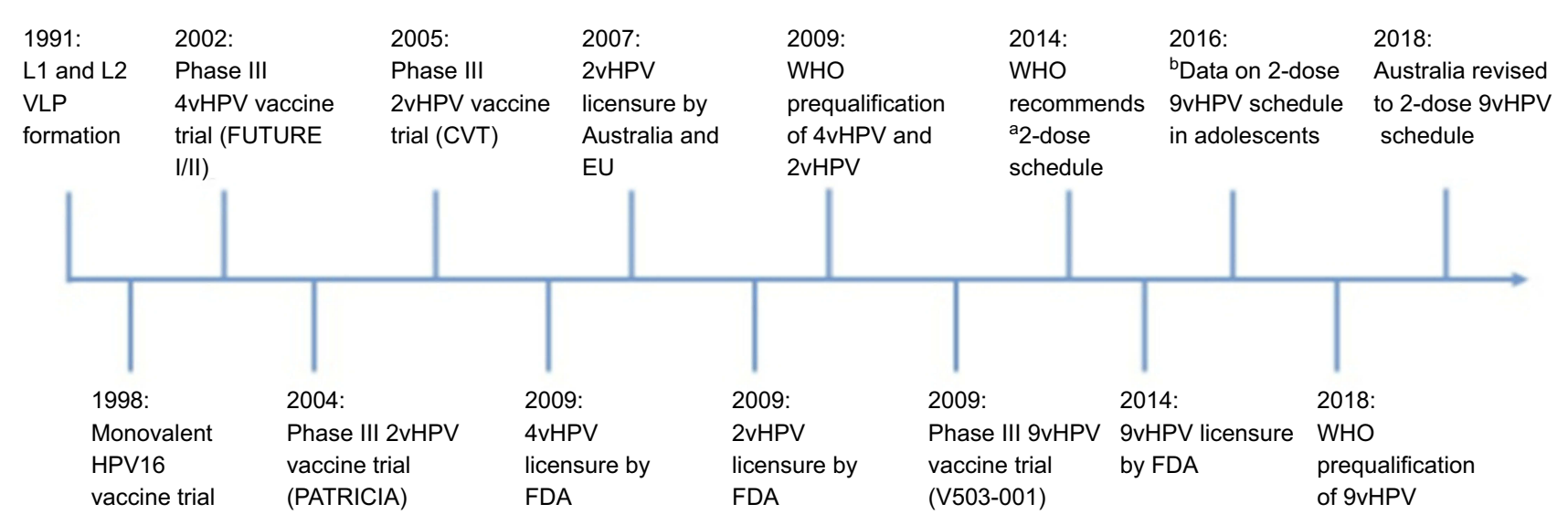

Figure I Timeline of pivotal Phase III HPV vaccine trials and licensure/registration of the HPV vaccines.

Notes: ${ }^{a}$ Two-dose schedule separated by six months to adolescents aged $<15$ years. ${ }^{\text {b }}$ Data from Iversen et al. ${ }^{57}$

Abbreviations: 4vHPV, quadrivalent HPV vaccine; 2vHPV, bivalent HPV vaccine; 9vHPV, nonavalent HPV vaccine; FDA, The U.S. Food and Drug Administration; HPV, human papillomavirus; EU, European Union; VLP, virus-like particle.

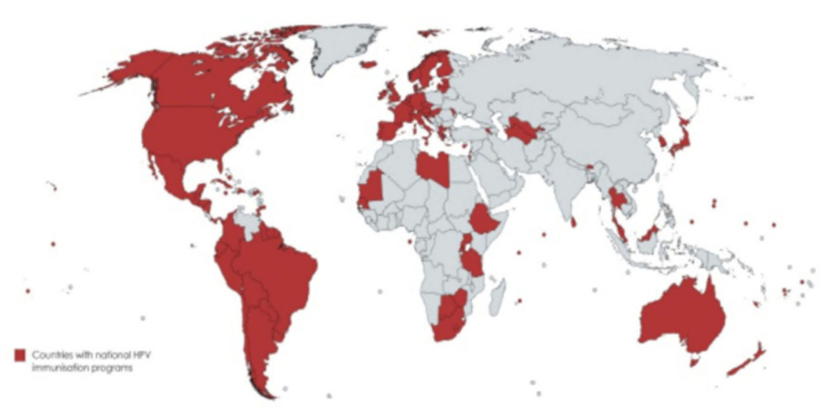

Figure 2 Countries that have introduced a national human papillomavirus immunization program using any of the three licensed vaccines (91 countries, $47 \%$ ). Source: Data from WHO. ${ }^{85}$

of cervical cancers. ${ }^{1}$ This review article summarizes the efficacy of 9vHPV in preventing HPV infection and related anogenital precancers as demonstrated in clinical trials to date, as well as the potential impact of this vaccine on the cervical cancer burden globally. We searched for studies and review articles that evaluated the safety, efficacy, and immunogenicity of 9vHPV using PubMed, Google Scholar, and scientific review/comment papers during August 2018-January 2019, and included them in this review article. Search terms used include "Gardasil9", "nine-valent HPV vaccine", "burden of cervical cancer", "HPV vaccine licensure", "HPV vaccination", "HPV vaccines", and "cost-effectiveness".

\section{Burden of HPV-associated diseases}

HPV infection is one of the most common sexually transmitted infections worldwide. ${ }^{2}$ A meta-analysis of over one million women from 194 studies found an overall HPV prevalence of approximately $12 \%$ in asymptomatic women globally. ${ }^{3}$ The highest HPV prevalence is in the sub-Saharan African regions (24\%), although these estimates vary by region, study design, as well as age of participants. ${ }^{3}$ Most individuals (approximately 90\%) infected with HPV are asymptomatic and are able to clear the infection through host immune responses within two years. ${ }^{4}$ However, for the small proportion who have persistent infection, this can lead to a range of diseases from benign lesions to anogenital cancers as well as some oropharyngeal diseases. There are currently more than 100 HPV genotypes identified that can be classified into high- and low-risk types depending on the potential neoplastic disease they cause.

Low-risk HPV types 6 and 11 cause 90\% of anogenital warts and benign/low-grade abnormalities in the genital areas. ${ }^{5,6}$ These HPV types are also responsible for causing recurrent respiratory papillomatosis in the respiratory tract. $^{7}$ The high-risk HPV types are known to cause anogenital cancers, which includethe majority of cervical cancer cases and a significant proportion of vaginal (78\%) and anal $(88 \%)$ cancers, as well as some vulvar $(25 \%)$ and penile $(50 \%)$ cancers. $^{8,9}$ In addition, high-risk types, in particular HPV16, are also responsible for an increasing number of oropharyngeal cancers (31\%) largely in the base of the tongue and tonsillar areas. ${ }^{10,11}$ These diseases are more common in immunocompromised individuals such as those infected with HIV or post transplantation. ${ }^{12}$

Cervical cancer remains the fourth most common cancer in women worldwide, with an estimated 570,000 cases and 
311,000 deaths annually. ${ }^{13}$ The majority of these cases occur in low-and lower-middle-income countries (LLMICs) due to multiple factors. These factors include the lack of comprehensive cervical cancer screening programs and treatment of lesions found on screening, no access to HPV vaccine due to the high vaccine cost, limited health services to deliver these vaccines, as well as the vaccine being seen as a low priority. ${ }^{14,15}$ Moreover, issues such as vaccine hesitancy, and social and cultural factors have also impeded the introduction of HPV vaccines. ${ }^{14}$ Globally, HPV16 and 18 are responsible for $70 \%$ of cervical cancer. ${ }^{9}$ Together with HPV types $31 / 33 / 45 / 52 / 58$, these nine types are responsible for approximately $90 \%$ of cervical cancers worldwide. ${ }^{8,99}$ The prevalence of these five HPV types varies geographically, with higher prevalence of HPV52 and 58 in East Asia and North America, while HPV31, 33 and 45 are more common in European countries. ${ }^{3,16}$

\section{First-generation HPV vaccines}

The formulations and schedules of 4vHPV and $2 \mathrm{vHPV}$ are summarized in Table 1. Although both vaccines have aluminum-based adjuvant, 2vHPV also contains a monophosphoryl lipid A adjuvant component, which activates innate immunity through Toll-like receptor $4 .{ }^{17}$ This adjuvant system is also known as AS04 and is thought to account for the higher immunogenicity induced by $2 \mathrm{vHPV}$ when compared with $4 \mathrm{vHPV}$, although the clinical significance remains unknown (there has been no breakthrough disease seen in those vaccinated and thus no defined immune correlate of protection). ${ }^{18,19}$ Both $4 \mathrm{vHPV}$ and $2 \mathrm{vHPV}$ have greater than $90 \%$ efficacy against cervical intraepithelial neoplasia grade 1-3, adenocarcinoma in situ, and invasive cervical carcinoma due to HPV16/18 in women aged 15-26 years. ${ }^{20-23}$ Although both vaccines might provide protection against other HPV-related cancers such as vulvar, vaginal, and anal, ${ }^{24}$ only $4 \mathrm{vHPV}$ is licensed against these cancers as well as against HPV6- and HPV11related genital warts.

Neutralizing antibodies are thought to be the primary mediator of protection for HPV vaccines, although to date, the actual level required for protection (ie, correlate) has not been identified. Overall, seroconversion occurs in $99-100 \%$ of those vaccinated in randomized double-blind placebocontrolled trials. ${ }^{25}$ Antibody responses following vaccination peak at month seven (one month after the third dose) at titers between 10- and 100-fold higher than the levels found following natural infection. ${ }^{25}$ Such antibody responses are found to persist at a level several-fold higher than natural infection for more than 10 years. ${ }^{26}$ The high immunogenicity of the HPV vaccine is thought to be due to the dense and ordered structure of the VLPs, as well as the route of administration in comparison to the natural infection route. ${ }^{27}$

The high vaccine efficacy observed in vaccinated young adults (age 16-26 years) not infected or previously infected with the vaccine HPV types has prompted the use of HPV vaccine in young adolescents before the onset of sexual activity. Immunobridging studies evaluating either $4 \mathrm{vHPV}$ or $2 \mathrm{vHPV}$ in adolescent girls/boys and women following a three-dose schedule found at least one- to twofold higher type-specific antibodies in the younger age group. ${ }^{28-30}$ Moreover, several studies then found that two doses given to adolescents six months apart had antibody responses that were non-inferior to women who were given the standard three-dose schedule, summarized by Toh et al. ${ }^{31}$ These studies have led the WHO to revise its recommendation in 2014 to include a two-dose HPV vaccine schedule for girls aged $<15$ years, provided the interval between each dose is at least six months. ${ }^{32}$

Both 2vHPV and 4vHPV also provide some degree of cross-protection to nonvaccine but phylogenetically related HPV types (ie, HPV31, 33, and 45), which is likely to be mediated by cross-neutralizing antibodies. ${ }^{33-38}$ In

Table I Characteristics of HPV VLP vaccines

\begin{tabular}{|c|c|c|c|}
\hline Manufacturer & Merck $^{\mathrm{TM}}\left(\right.$ Gardasil $\left.^{\circledR}\right)$ & GlaxoSmithKline $^{\mathrm{TM}}$ (Cervarix $^{\circledR}$ ) & Merck $^{\mathrm{TM}}$ (Gardasil ${ }^{\circledR}$ 9) \\
\hline LI VLP types & $6,11,16$, and 18 & 16 and 18 & $6, \mathrm{II}, \mathrm{I} 6, \mathrm{I} 8,3 \mathrm{I}, 33,45,52$, and 58 \\
\hline Dose & $20 / 40 / 40 / 20 \mu g$ & $20 / 20 \mu g$ & $30 / 40 / 60 / 40 / 20 / 20 / 20 / 20 / 20 \mu \mathrm{g}$ \\
\hline Producer cells & $\begin{array}{l}\text { Saccharomyces cerevisiae (baker's } \\
\text { yeast) expressing LI }\end{array}$ & $\begin{array}{l}\text { Trichoplusia ni }(\mathrm{Hi} 5) \text { insect cell line infected with } \\
\text { LI recombinant baculovirus }\end{array}$ & $\begin{array}{l}\text { Saccharomyces cerevisiae (baker's } \\
\text { yeast) expressing LI }\end{array}$ \\
\hline Adjuvant & $\begin{array}{l}225 \mu g \text { aluminium hydroxypho- } \\
\text { sphate sulfate }\end{array}$ & $\begin{array}{l}500 \mu \mathrm{g} \text { aluminium hydroxide, } 50 \mu \mathrm{g} \\
\text { 3-O-deacylated-4'-monophosphoryl lipid A }\end{array}$ & $\begin{array}{l}500 \mu g \text { aluminium hydroxypho- } \\
\text { sphate sulfate }\end{array}$ \\
\hline Vaccination schedule & 0,2 , and 6 months & $0, \mathrm{I}$, and 6 months & 0,2 , and 6 months \\
\hline
\end{tabular}

Abbreviations: HPV, human papillomavirus; VLP, virus-like particle. 
most cases, 2vHPV generated significantly higher neutralizing antibodies for HPV31 and 45 than 4vHPV, possibly due to its unique adjuvant properties, although the clinical significance of this is unknown. ${ }^{37,39,40}$ These crossneutralizing antibodies are approximately 100 -fold lower than vaccine-type antibodies, ${ }^{41}$ which raises the question of the duration of protection against these nonvaccine types.

\section{Vaccine efficacy of 9vHPV}

The appeal of $9 \mathrm{vHPV}$ is the potential to protect against the nine most common cancer-causing HPV types, as well as the majority of genital warts cases. Since 9vHPV was only licensed in the last five years and rollout in public health programs is only recent, population data are still several years away. Phase III studies have demonstrated that 9vHPV is safe and highly efficacious against HPV infection and anogenital precancer lesions in both men and women (Table 2). Of note, the pivotal trial that led to the licensure of 9vHPV was an international multicenter, double-blind randomized controlled trial of more than 10,000 women aged 16-26 years. The study reported greater than 96\% vaccine efficacy against high-grade cervical, vulvar, or vaginal disease as well as six-month persistent infection caused by HPV $31,33,45,52$, and 58 in women not previously infected with HPV following three doses of $9 \mathrm{vHPV}^{42}$ This high efficacy (90-98\%) of 9vHPV in preventing certain HPV-related precancers was sustained for up to six years. ${ }^{43}$ Due to ethical concerns, 9vHPV was compared with $4 \mathrm{vHPV}$ instead of a placebo control in this study. The incidence of disease related to HPV-6, 11, 16, and 18 was similar between both groups, with comparable antibody responses observed, suggesting that $9 \mathrm{vHPV}$ is just as effective as $4 \mathrm{vHPV}$ for these types. In addition, all participants who received 9vHPV seroconverted to the additional five HPV types (HPV31, 33, 45, 52 and 58) one month following the last dose, and the levels of these five additional HPV types were significantly higher than in $4 \mathrm{vHPV}$ recipients.

The burden of cervical cancer and risk is different across populations around the world. Subgroup analyses from two Phase III double-blind randomized controlled trials were performed among Asian and Latin American participants to determine the population-specific vaccine efficacy. ${ }^{44,45}$ Similar high vaccine efficacy (>96\%) against any grade of cervical, vulvar, and vaginal diseases, as well as greater than 93\% against six-month persistent HPV infection, were found for both ethnic populations in individuals who were not infected with HPV. These findings were consistent with the vaccine efficacy globally, suggesting that $9 \mathrm{vHPV}$ is as efficacious in Asia and Latin America.

\section{Reactogenicity and immunogenicity of 9vHPV}

Antibody responses to 9vHPV types and the comparison of the shared HPV genotypes (HPV6, 11, 16, and 18) between 9vHPV and 4vHPV were investigated in clinical studies consisting of young women (aged 16-26 years) and young adolescent boys and girls (aged 9-15 years) (Table 3). ${ }^{46-49}$ Almost $100 \%$ of individuals who received 9vHPV seroconverted to all vaccine types one month following the third dose, and the antibody levels for HPV6, 11, 16, and 18 were similar between the two vaccine groups, suggesting that the addition of five new types to 9vHPV did not result in interference and hence did not alter immunogenicity to the $4 \mathrm{vHPV}$ types. ${ }^{42,50}$ Prior to 9vHPV licensure, three immunobridging Phase II studies evaluated the vaccine dose formulation to ensure the optimal formulation in terms of immunogenicity and safety of a multivalent HPV vaccine. ${ }^{51}$

Around $77.5-100 \%$ of individuals who received three doses of 9vHPV remained seropositive to all 9vHPV after five years. ${ }^{43}$ When a fourth dose of 9vHPV was given to this group of individuals, antibody responses were 1.25-4.10- and 1.65-4.88-fold higher at one week and one month after the fourth dose, respectively, when compared to the levels at one month after the third dose, suggesting the induction of immunological memory to all nine HPV types following the three-dose primary series. ${ }^{52}$ Higher antibody responses to all 9vHPV types were observed in young adolescents when compared with young adults, consistent with the earlier findings for $4 \mathrm{vHPV}$ and $2 \mathrm{vHPV}^{50}$ Between male and females, the immune responses were non-inferior at one month after the third dose, although the geometric mean titers to all nine HPV types were numerically lower in men who have sex with men and in women than in heterosexual men; geometric mean titers were lower among men who have sex with men compared to women, which may be due to the higher preexposure levels in these groups prior to vaccination. $^{53}$

9vHPV is also immunogenic to all nine HPV types in young women previously vaccinated with three doses of $4 \mathrm{vHPV} .{ }^{54}$ However, it is interesting to note that women 


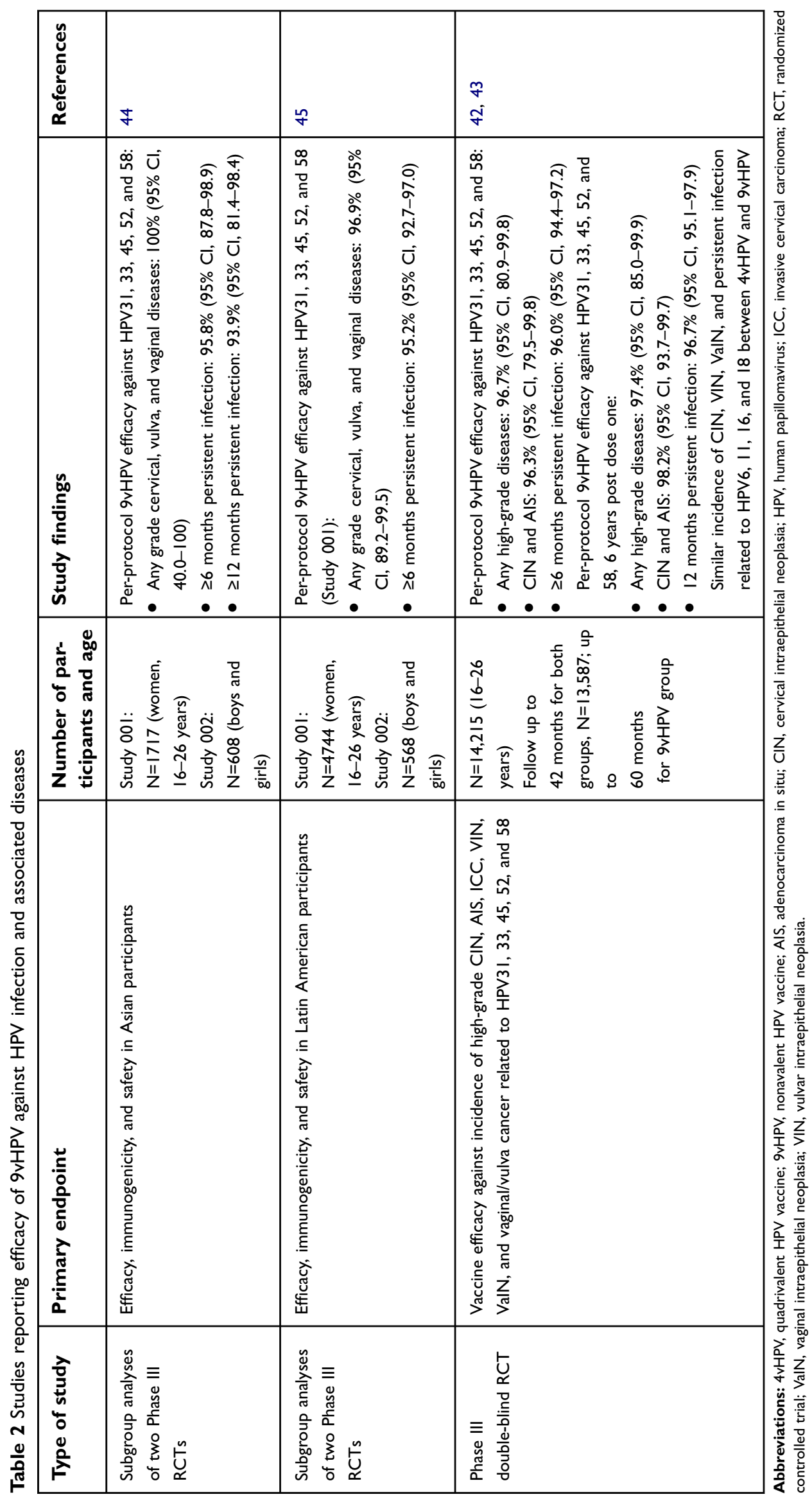




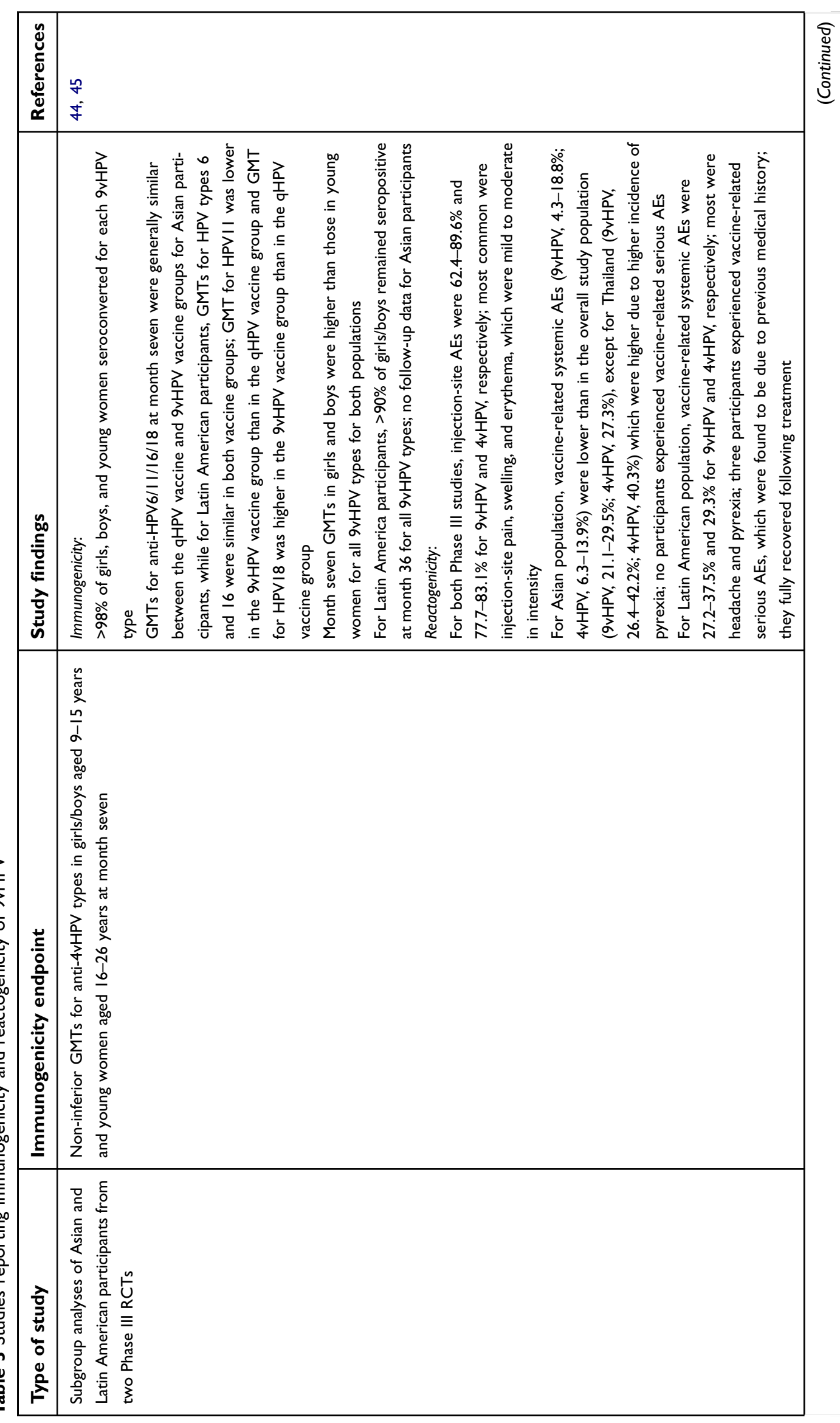




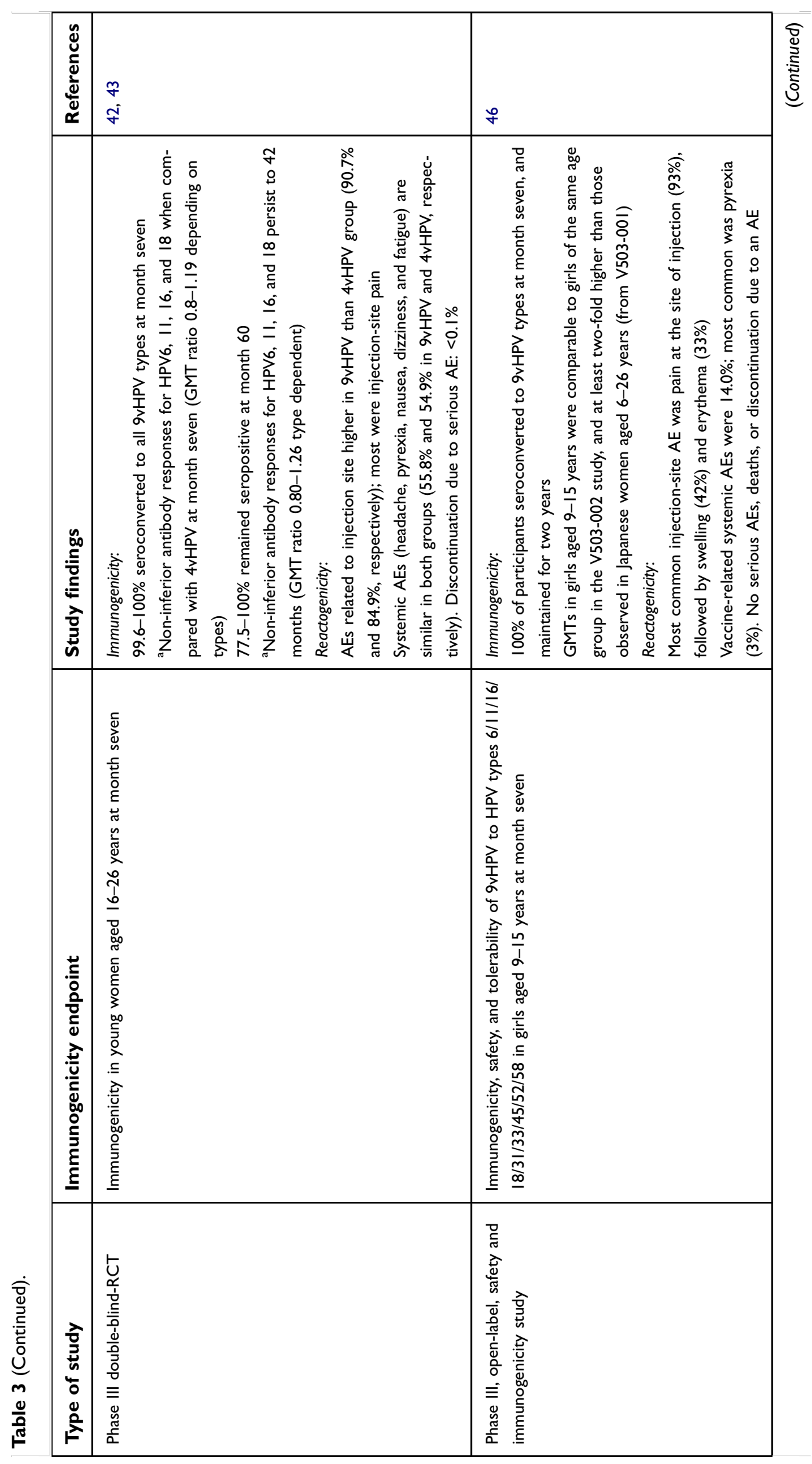




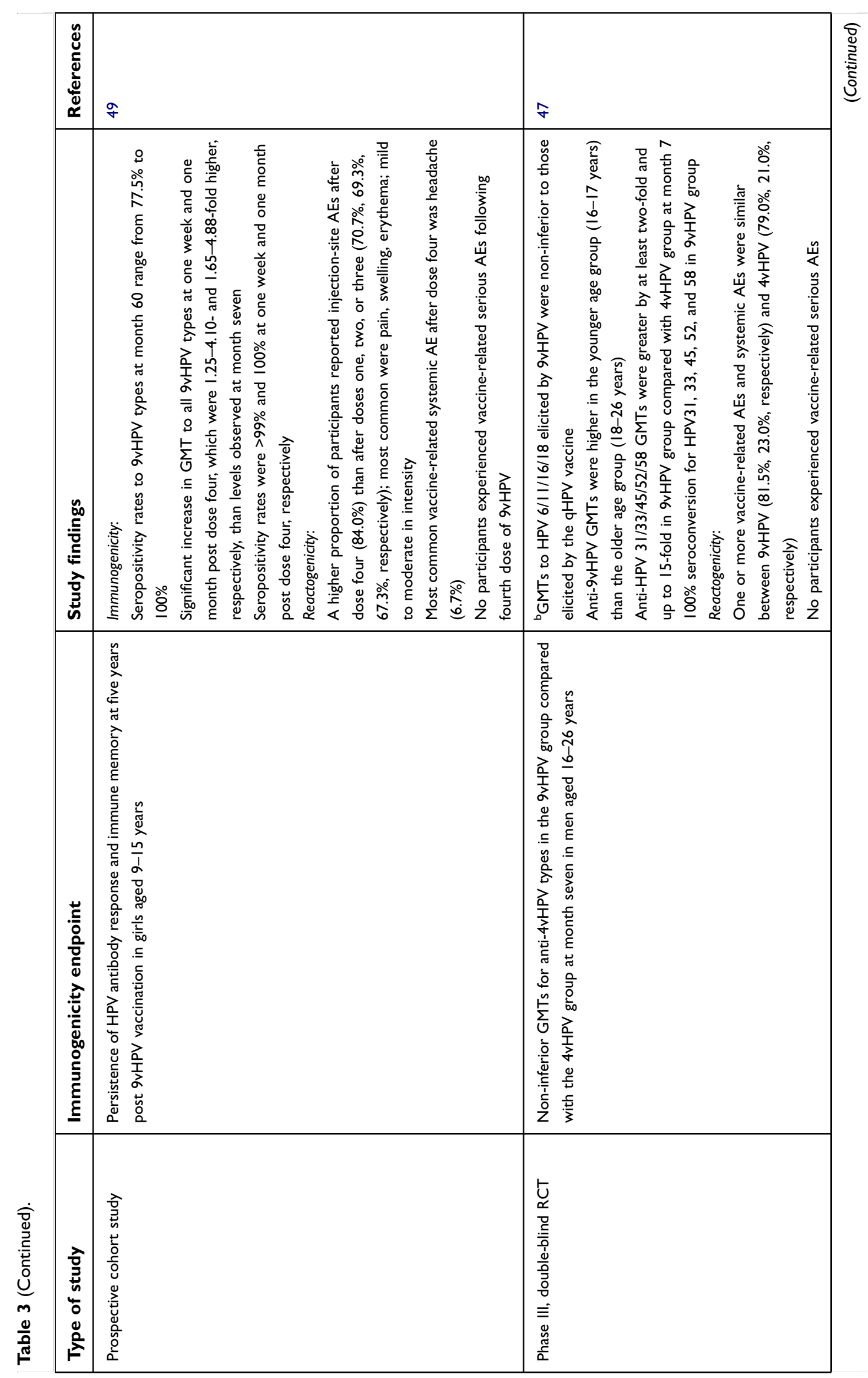




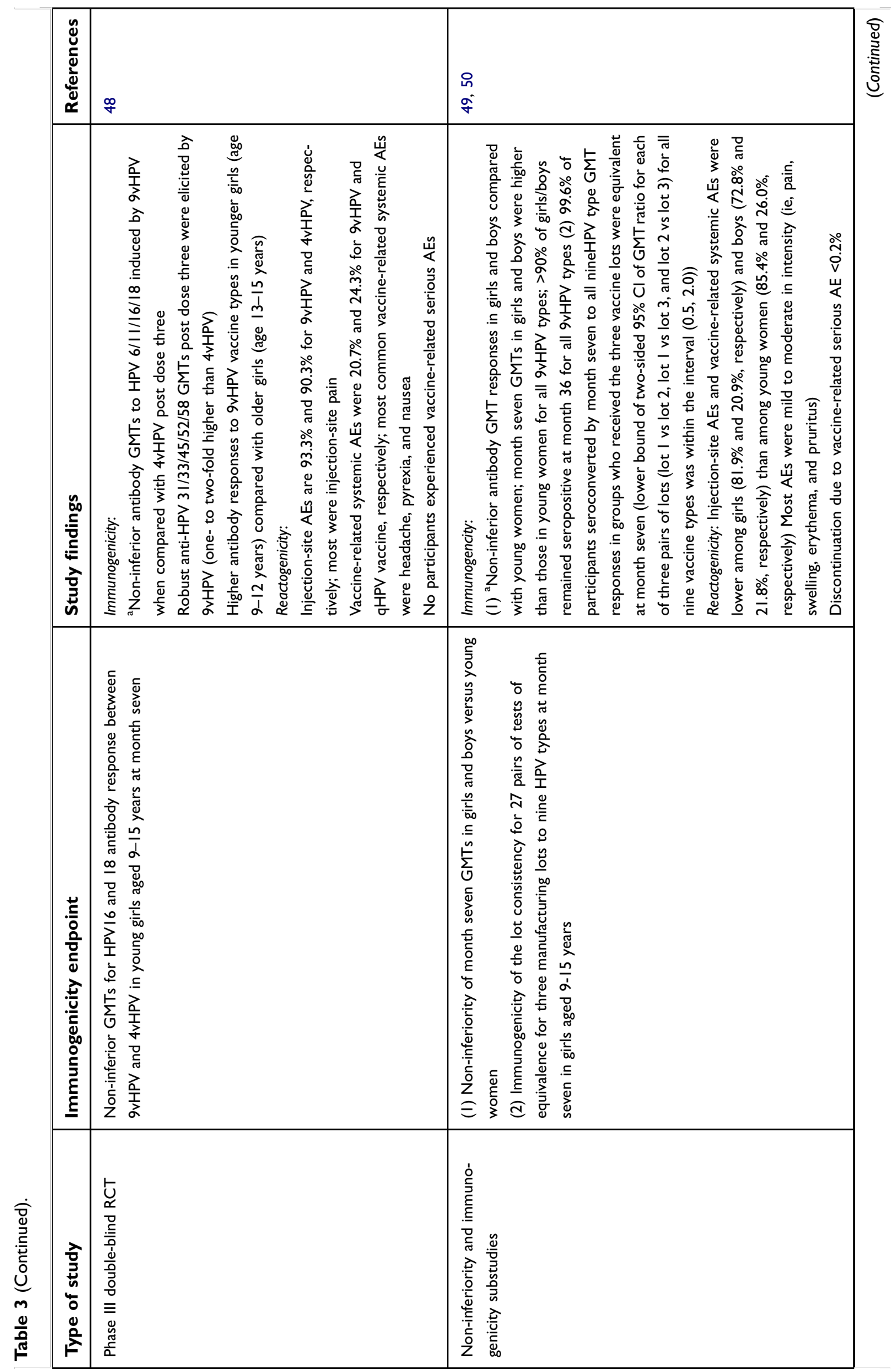




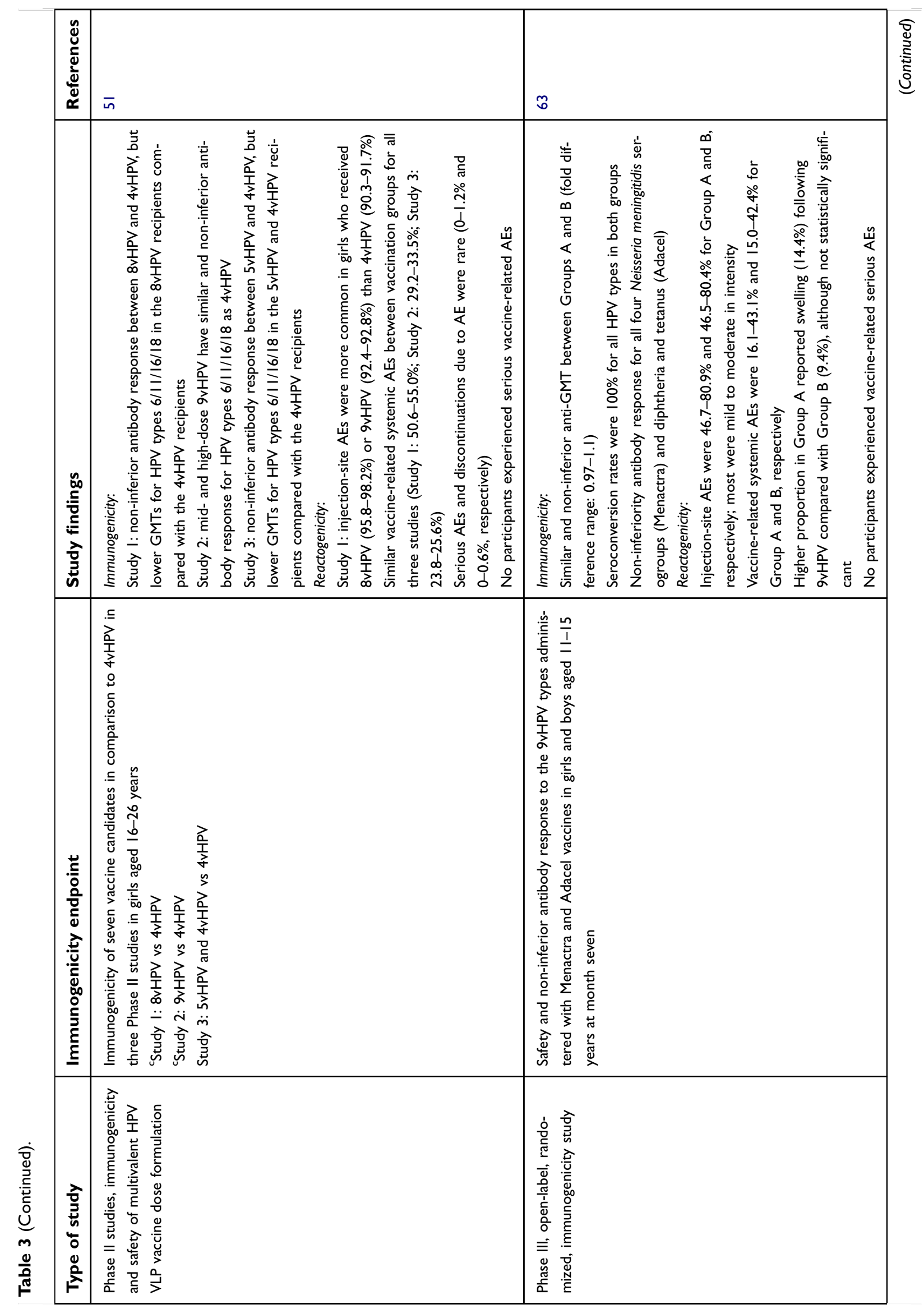




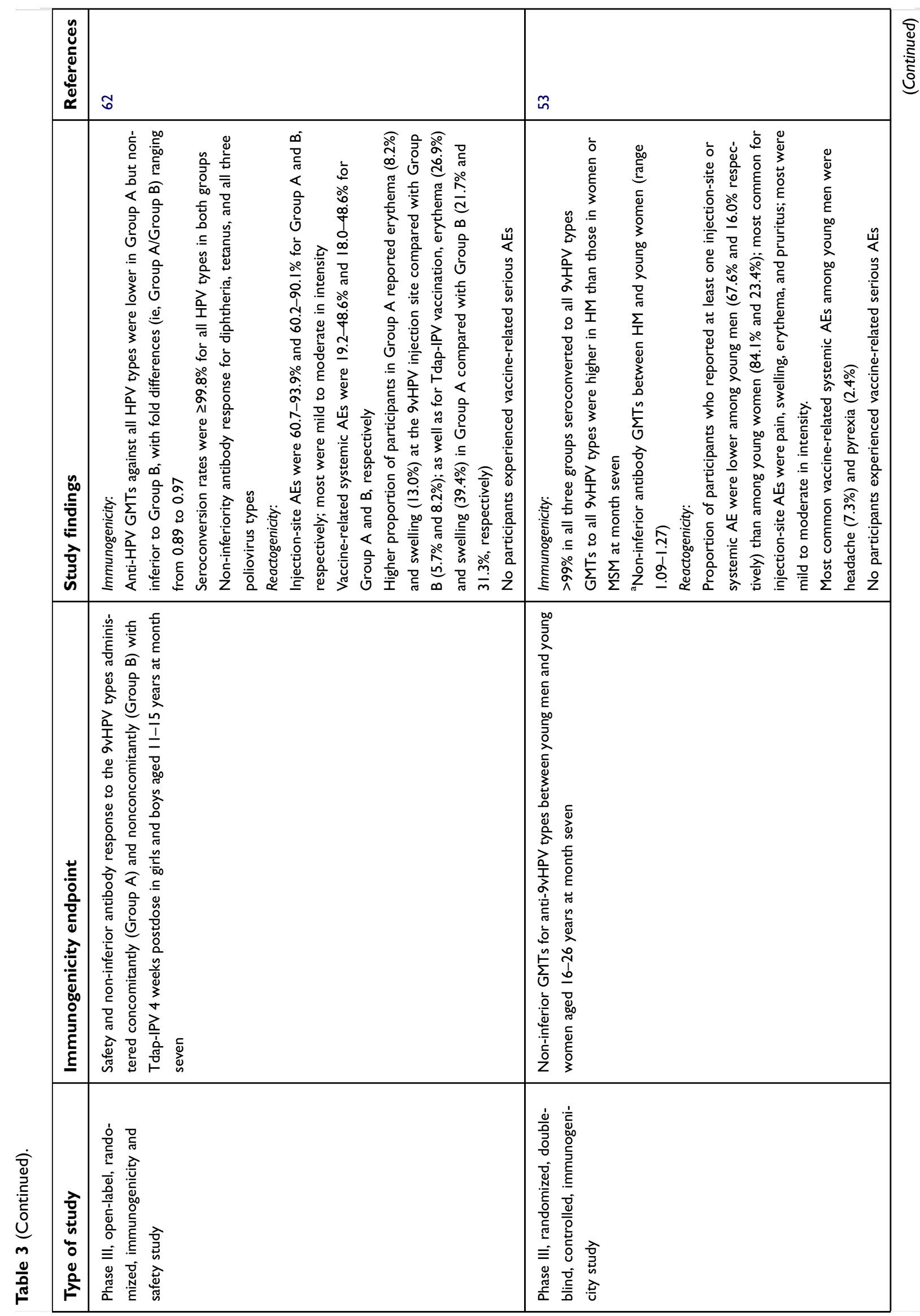




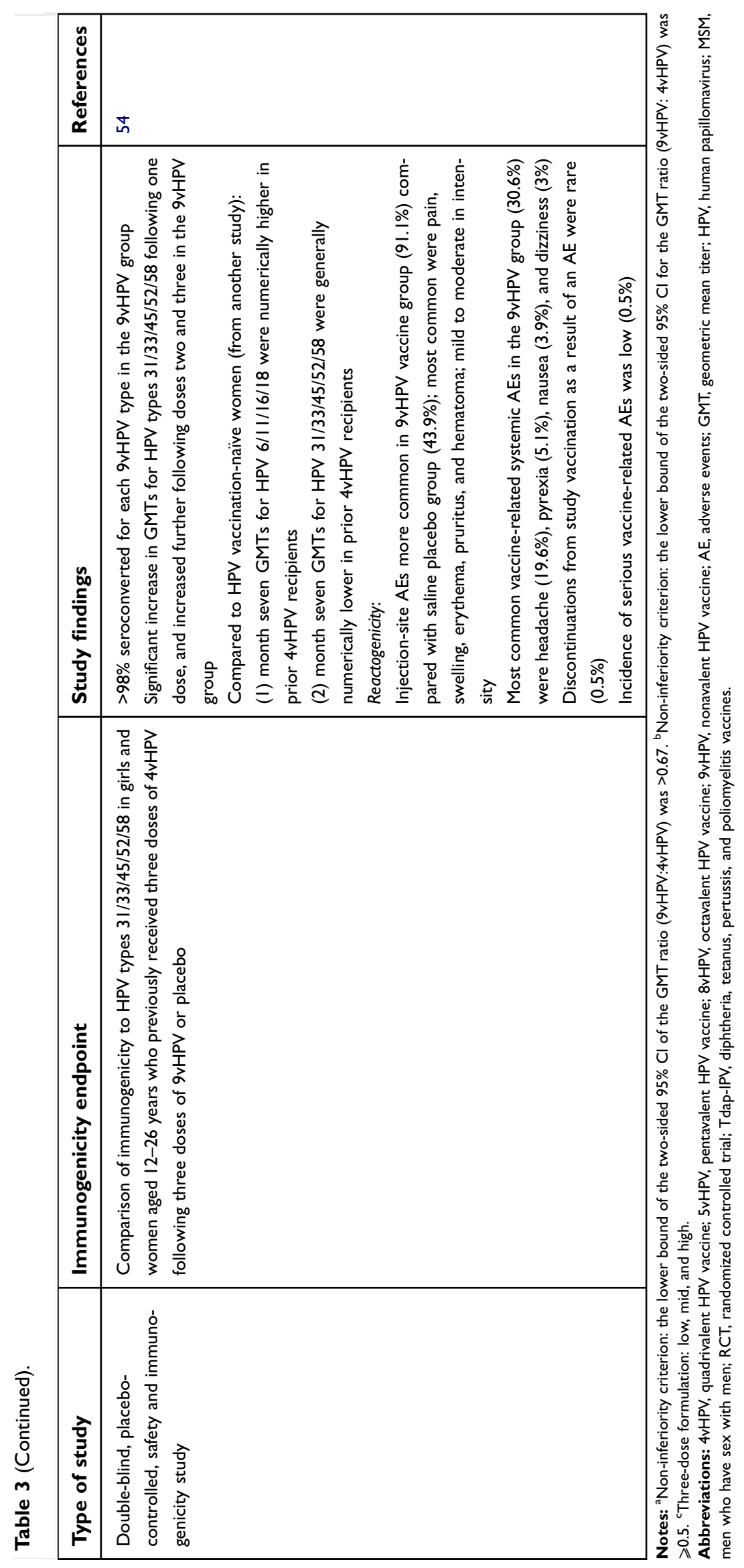


who were naïve to any HPV vaccination and received three doses of $9 \mathrm{vHPV}$ had higher antibody responses to HPV31/33/45/52/58 when compared with women who previously received three doses of $4 \mathrm{vHPV}$ (who were seronegative to HPV31/33/45/52/58 after 12-36 months) and received three doses of 9vHPV. Several mechanisms have been proposed for this observation, including preferential activation of cross-reactive memory cells compared to naïve cells and/or preferential activation of memory response to HPV 16/18 L1 proteins, resulting in a blunting of primary responses to antigenically related HPV31/33/45/52/58 L1 proteins (the theory of original antigenic sin; the immune system activates the memory responses of the earlier infection/vaccination when the epitope varies slightly, inducing faster and stronger responses, rather than mounting another primary or secondary response to the new epitope). ${ }^{55}$ The clinical significance of this lower immune response to HPV31/33/45/ $52 / 58$ following 9 vHPV vaccination in women previously vaccinated with $4 \mathrm{vHPV}$ is unknown. Nevertheless, the antibody level to these types was still several-fold higher than natural infection and the study demonstrated that it was safe to give 9vHPV to individuals previously vaccinated with $4 \mathrm{vHPV}$ or $2 \mathrm{vHPV}$. Therefore, individuals who wish to get additional protection to the five new types may still require the standard two- or three-dose 9vHPV schedule depending on their age and duration between doses. ${ }^{56}$

Similar to the findings of the $4 \mathrm{vHPV}$ and $2 \mathrm{vHPV}$ noninferiority studies, two doses of 9vHPV given six or 12 months apart to adolescent boys and girls (age 9-14 years) generated non-inferior antibody responses (to all nine HPV types) when compared with young women aged 16-26 years who received three doses, one month after the last dose. ${ }^{57}$ This study was the basis of U.S. FDA approval for a two-dose 9vHPV schedule to girls and boys younger than age 15 years, including recommendations by the USA, European countries, and Australia. ${ }^{58-60}$

To date, more than 20,000 individuals have received three doses of 9vHPV in Phase II and III clinical trials (reactogenicity data summarized in Table 3). 9vHPV is shown to be safe and generally well tolerated in participants aged 9-26 years, with a similar adverse event profile to that of 4vHPV (which is used in many countries globally as part of national immunization programs). ${ }^{61}$ The most common adverse event from seven Phase III clinical trials was injection-site pain, swelling, and redness, which was more common for 9vHPV than 4vHPV with increasing number of doses. ${ }^{61}$ It is important to note that the adjuvant content in 9vHPV is more than double that of 4vHPV (0.5 vs $0.225 \mathrm{mg}$ ), and also has a higher VLP antigen content (refer to Table 1). Nevertheless, most adverse events were mild to moderate in intensity. ${ }^{61}$ Discontinuation due to adverse events and serious vaccinerelated adverse events in the clinical trials was rare. ${ }^{61}$ There were three reports of vaccine-related serious adverse events, although the participants were later found to have preexisting medical conditions when receiving the vaccine. A 10-year-old boy who has a previous history of seasonal allergy and bronchial asthma experienced an asthma exacerbation one day after receiving the first 9vHPV dose. ${ }^{50}$ The boy was hospitalized, but fully recovered the following day. Another participant (age 21 years) from the same study experienced a severe headache on the day she received the third 9vHPV dose and was hospitalized the next day with neck stiffness, headache, and fever $\left(39.0^{\circ} \mathrm{C}\right)$. She was discharged from the hospital two days after receiving symptomatic treatment. It was noted that she was bitten by a spider five days before the third dose and was still undergoing treatment when she received the vaccine. ${ }^{50}$ In another study, one participant (age 18 years) was hospitalized for tonsillitis two days after receiving the first dose of 9vHPV vaccine, but fully recovered following incision and drainage of the tonsils as well as antibiotic treatment. ${ }^{54}$

Coadministration of 9vHPV and other adolescent vaccines (ie, Neisseria meningitidis serotypes A/C/Y/W-135, diphtheria/tetanus/acellular pertussis, or diphtheria/tetanus/ acellular pertussis/inactivated poliomyelitis vaccine) to boys and girls aged 9-14 years was also found to be safe and immunogenic when compared with those who received the vaccines nonconcomitantly. ${ }^{62,63}$ Together, these data support the high safety profile of this vaccine, as well as the feasibility of concomitant administration of 9vHPV with common adolescent vaccines as a public health strategy.

\section{Population effect and future directions}

A long-term follow-up study to assess the effectiveness of $9 \mathrm{vHPV}$ for at least 14 years in participants in Scandinavian countries (ie, Denmark, Norway, and Sweden) from the Phase III 9vHPV efficacy study (V503-001) is currently ongoing. ${ }^{64}$ The incidence of cervical precancers and cancers due to the seven oncogenic types in the vaccine (HPV 16/18/31/33/45/52/58) will be 
compared to the estimated incidence rate in an unvaccinated cohort of similar age and risk level. This information will be critical for the longevity of protection offered by this vaccine.

9vHPV has been licensed in a number of regions including Australia (Therapeutic Goods Association), the USA (FDA), Canada (Health Canada), the European Union (European Medicines Agency), and a number of countries in Asia such as China and Korea. To our knowledge, Australia, Canada, and the USA are using 9vHPV in their national immunization programs, and while both Australia and Canada have schoolbased programs, HPV vaccination is offered through primary care providers in clinic-based settings in the USA. ${ }^{65-67}$ Australia recently revised their HPV vaccination schedule in February 2018 from three doses of 4vHPV to two doses of $9 \mathrm{vHPV}$ in adolescent boys and girls aged 12-13 years. Australia was one of the first countries to introduce a government-funded school-based HPV vaccine program (4vHPV in 2007) with demonstrated high vaccine coverage (around 80\% for three doses) in women aged $<18$ years and is associated with a significant decrease of $0.38 \%$ in high-grade cervical abnormalities within three years of vaccine introduction. $^{68}$ The prevalence of high-risk vaccine HPV type declined from $22 \%$ in the prevaccine era to $1.5 \%$ among girls aged 18-24 years, nine years after vaccine introduction. $^{69,70}$ It was postulated that the replacement of 4vHPV with 9vHPV in Australia can potentially protect against an additional $15 \%$ and $11 \%$ of cervical cancer and anal cancers, respectively. ${ }^{71}$ The impact of 9vHPV and the HPV prevalence in Australia can be evaluated by "a built in approach" which utilizes the genotyping from screening using HPV nucleic acid testing, which was recently introduced to replace Pap screening (largely due to the effects of HPV vaccination on Pap abnormalities and the introduction of a more sensitive and objective test). ${ }^{72}$ With the use of 9vHPV coupled with high vaccine coverage in a genderneutral vaccination program, and robust HPV screening, Australia is likely to be the first country to eliminate cervical cancer (defined as four new cases per 100,000 women each year) by $2028 .^{73}$ The incidence of cervical cancer is expected to further decrease to less than one case per 100,000 women by $2066 .^{73}$ The national HPV vaccine schedule in Quebec, Canada is unique, consisting of one dose of 9vHPV followed by a dose of 2vHPV six months later to nine-year-old girls and boys; mixed HPV vaccine schedule. The potential cost-savings from this mixed schedule based on the lower cost for 2vHPV are approximately $\$ 3$ million per vaccinated birth cohort compared with a two-dose program with 9vHPV. These savings could be used for catch-up vaccination in older age groups, representing an attractive vaccination strategy, provided it is safe and equivalent protection can be achieved. ${ }^{74}$ The HPV vaccines are safe, and have similar safety profiles between the mixed vaccine schedule and the standard schedule as demonstrated in several cohort studies. ${ }^{54,75-77}$ In addition, higher immunogenicity to HPV16 and 18 (possibly due to the unique adjuvant in 2vHPV) and lower immunogenicity to the HPV types that are not in 2vHPV are observed in the mixed schedule when compared with the standard 9vHPV schedule. ${ }^{75}$ This finding is not unexpected although the clinical significance is unknown. Therefore, long-term follow up including the prevalence of genital warts and other HPVrelated diseases as well as circulating HPV types following such schedules are extremely important, and studies are in place to monitor these outcomes. ${ }^{74}$

Several studies have found the use of 9vHPV to be highly cost effective in high-income countries when the vaccine is given to both boys and girls, despite the higher costs associated with the use of 9vHPV compared with $2 \mathrm{vHPV}$ or $4 \mathrm{vHPV}{ }^{78-81}$ Although these results are largely dependent on the vaccine cost, they were generally robust across varying assumptions based on the burden of disease, vaccine coverage, and duration of protection including crossprotection of $2 \mathrm{vHPV}$ or $4 \mathrm{vHPV}$, as well as health care costs within high-income countries. It is important to note that the majority of the HPV-associated cancers, particularly cervical cancer, occurs in LLMICs, and the use of HPV vaccine is inversely proportionate to countries with the highest disease burden; only $1.7 \%$ of women in LLMICs are estimated to have received one dose of any HPV vaccine. ${ }^{82}$ The use of 9vHPV in LLMICs in the foreseeable future is unlikely due to its high cost compared to either $2 \mathrm{vHPV}$ or $4 \mathrm{vHPV}$. However, ongoing studies are underway to examine the efficacy and immunogenicity of a single-dose HPV vaccine (9vHPV and 2vHPV) in Costa Rica and Tanzania, respectively, ${ }^{83,84}$ which may be a "game-changer" for LLMICs. The continued investigation of novel strategies such as single-dose schedules, or mixed HPV vaccine schedules, will be important in the continued use and implementation of HPV vaccine in high burden settings.

\section{Conclusion}

$9 \mathrm{vHPV}$ is safe and offers the potential to prevent $90 \%$ of cervical cancers and a significant proportion of HPVrelated vulvar, vaginal, and anal cancers. In high-income countries that have high vaccine coverage and a robust HPV screening program, the transition from $2 \mathrm{vHPV}$ or 
4vHPV to 9vHPV as a national immunization program will likely further decrease the incidence of HPV-associated diseases and the costs associated with cancer treatment due to the additional cancer-causing types in the vaccine. As for LLMICs, any HPV vaccine use in a national program (demonstrated to have the highest coverage) is the most feasible option with maximum impact on cervical cancer morbidity and mortality. Financial and logistical support in these countries are crucial, which will be alleviated with a reduced-dose schedule, particularly a singledose schedule if proven effective.

\section{Disclosure}

FMR and PVL are recipients of National Health and Medical Research Council Fellowships. SMG has received grants through her institution from Merck, GlaxoSmithKline, CSL, and the Commonwealth Department of Health; and has delivered lectures and received speaking fees from MSD for work performed in her personal time. SMG is also a member of Global Advisory Board HPV. The authors report no other conflicts of interest in this work.

\section{References}

1. Brotherton JML, Tabrizi SN, Phillips S, et al. Looking beyond human papillomavirus (HPV) genotype 16 and 18: defining HPV genotype distribution in cervical cancers in Australia prior to vaccination. Int J Cancer. 2017;141(8):1576-1584. doi:10.1002/ijc.30871

2. Chesson HW, Dunne EF, Hariri S, Markowitz LE. The estimated lifetime probability of acquiring human papillomavirus in the United States. Sex Transm Dis. 2014;41(11):660-664. doi:10.1097/ OLQ.0000000000000193

3. Bruni L, Diaz M, Castellsague X, Ferrer E, Bosch FX, de Sanjose S. Cervical human papillomavirus prevalence in 5 continents: meta-analysis of 1 million women with normal cytological findings. J Infect Dis. 2010;202(12):1789-1799. doi:10.1086/657321

4. Molano M, Van den Brule A, Plummer M, et al. Determinants of clearance of human papillomavirus infections in Colombian women with normal cytology: a population-based, 5-year follow-up study. $\mathrm{Am}$ J Epidemiol. 2003;158(5):486-494. doi:10.1093/aje/kwg171

5. Garland SM, Steben M, Sings HL, et al. Natural history of genital warts: analysis of the placebo arm of 2 randomized phase III trials of a quadrivalent human papillomavirus (types $6,11,16$, and 18) vaccine. J Infect Dis. 2009;199(6):805-814. doi:10.1086/597071

6. Patel H, Wagner M, Singhal P, Kothari S. Systematic review of the incidence and prevalence of genital warts. BMC Infect Dis. 2013;13:39. doi:10.1186/1471-2334-13-39

7. Larson DA, Derkay CS. Epidemiology of recurrent respiratory papillomatosis. APMIS. 2010;118(6-7):450-454. doi:10.1111/j.16000463.2010.02619.x

8. de Martel C, Plummer M, Vignat J, Franceschi S. Worldwide burden of cancer attributable to HPV by site, country and HPV type. Int $J$ Cancer. 2017;141(4):664-670. doi:10.1002/ijc.30716

9. de Sanjose S, Quint WG, Alemany L, et al. Human papillomavirus genotype attribution in invasive cervical cancer: a retrospective cross-sectional worldwide study. Lancet Oncol. 2010;11 (11):1048-1056. doi:10.1016/S1470-2045(10)70230-8
10. D'Souza G, Kreimer AR, Viscidi R, et al. Case-control study of human papillomavirus and oropharyngeal cancer. $N$ Engl $J$ Med. 2007;356(19):1944-1956. doi:10.1056/NEJMoa065497

11. Marur S, D'Souza G, Westra WH, Forastiere AA. HPV-associated head and neck cancer: a virus-related cancer epidemic. Lancet Oncol. 2010;11(8):781-789. doi:10.1016/S1470-2045(10)70017-6

12. Frisch M, Biggar RJ, Goedert JJ. Human papillomavirus-associated cancers in patients with human immunodeficiency virus infection and acquired immunodeficiency syndrome. J Natl Cancer Inst. 2000;92 (18):1500-1510. doi:10.1093/jnci/92.18.1500

13. Bray F, Ferlay J, Soerjomataram I, Siegel RL, Torre LA, Jemal A. Global cancer statistics 2018: GLOBOCAN estimates of incidence and mortality worldwide for 36 cancers in 185 countries. CA Cancer J Clin. 2018. doi:10.3322/caac.21492

14. Toh ZQ, Licciardi PV, Russell FM, Garland SM, Batmunkh T, Mulholland EK. Cervical cancer prevention through HPV vaccination in low- and middle-income countries in Asia. Asian Pac J Cancer Prev. 2017;18(9):2339-2343. doi:10.22034/APJCP.2017.18.9.2339

15. Howard N, Gallagher KE, Mounier-Jack S, et al. What works for human papillomavirus vaccine introduction in low and middle-income countries? Papillomavirus Res. 2017;4:22-25. doi:10.1016/j.pvr.2017.06.003

16. Bruni L, Barrionuevo-Rosas L, Albero GAM, et al. Human papillomavirus and related diseases in Asia. Summary report. ICO Information Centre on HPV and Cancer (HPV Information Centre); 2018. Accessed April 17, 2015.

17. Garcon N, Wettendorff M, Van Mechelen M. Role of AS04 in human papillomavirus vaccine: mode of action and clinical profile. Expert Opin Biol Ther. 2011;11(5):667-677. doi:10.1517/14712598.2011.573624

18. Einstein MH, Baron M, Levin MJ, et al. Comparison of the immunogenicity and safety of Cervarix and Gardasil human papillomavirus (HPV) cervical cancer vaccines in healthy women aged 18-45 years. Hum Vaccin. 2009;5(10):705-719. doi:10.4161/hv.5.10.9518

19. Einstein MH, Levin MJ, Chatterjee A, et al. Comparative humoral and cellular immunogenicity and safety of human papillomavirus (HPV)-16/18 AS04-adjuvanted vaccine and HPV-6/11/16/18 vaccine in healthy women aged 18-45 years: follow-up through Month 48 in a Phase III randomized study. Hum Vaccin Immunother. 2014;10 (12):3455-3465. doi:10.4161/hv.36117

20. Future II Study Group. Quadrivalent vaccine against human papillomavirus to prevent high-grade cervical lesions. $N$ Engl J Med. 2007;356(19):1915-1927. doi:10.1056/NEJMoa061741

21. Garland SM, Hernandez-Avila M, Wheeler CM, et al. Quadrivalent vaccine against human papillomavirus to prevent anogenital diseases. N Engl J Med. 2007;356(19):1928-1943. doi:10.1056/NEJMoa061760

22. Dillner J, Kjaer SK, Future I/II Study Group, et al. Four year efficacy of prophylactic human papillomavirus quadrivalent vaccine against low grade cervical, vulvar, and vaginal intraepithelial neoplasia and anogenital warts: randomised controlled trial. BMJ. 341;2010:c3493. doi:10.1136/bmj.c3493

23. Lehtinen M, Paavonen J, Wheeler CM, et al. Overall efficacy of HPV-16/18 AS04-adjuvanted vaccine against grade 3 or greater cervical intraepithelial neoplasia: 4-year end-of-study analysis of the randomised, double-blind PATRICIA trial. Lancet Oncol. 2012;13 (1):89-99. doi:10.1016/S1470-2045(11)70286-8

24. Mensah FA, Mehta MR, Lewis JS Jr., Lockhart AC. The human papillomavirus vaccine: current perspective and future role in prevention and treatment of anal intraepithelial neoplasia and anal cancer. Oncologist. 2016;21(4):453-460. doi:10.1634/theoncologist.2015-0075

25. Harper DM, DeMars LR. HPV vaccines - A review of the first decade. Gynecol Oncol. 2017;146(1):196-204. doi:10.1016/j. ygyno.2017.04.004

26. Schwarz TF, Galaj A, Spaczynski M, et al. Ten-year immune persistence and safety of the HPV-16/18 AS04-adjuvanted vaccine in females vaccinated at 15-55 years of age. Cancer Med. 2017;6 (11):2723-2731. doi:10.1002/cam4.1155 
27. Schiller J, Lowy D. Explanations for the high potency of HPV prophylactic vaccines. Vaccine. 2018;36(32Pt A):4768-4773. doi:10.1016/j.vaccine.2017.12.079

28. Block SL, Nolan T, Sattler C, et al. Comparison of the immunogenicity and reactogenicity of a prophylactic quadrivalent human papillomavirus (types 6, 11, 16, and 18) L1 virus-like particle vaccine in male and female adolescents and young adult women. Pediatrics. 2006;118(5):2135-2145. doi:10.1542/peds.2006-0461

29. Pedersen C, Petaja T, Strauss G, et al. Immunization of early adolescent females with human papillomavirus type 16 and $18 \mathrm{~L} 1$ virus-like particle vaccine containing AS04 adjuvant. J Adolesc Health. 2007;40(6):564-571. doi:10.1016/j.jadohealth.2007.02.015

30. Petaja $T$, Keranen H, Karppa T, et al. Immunogenicity and safety of human papillomavirus (HPV)-16/18 AS04-adjuvanted vaccine in healthy boys aged 10-18 years. $J$ Adolesc Health. 2009;44 (1):33-40. doi:10.1016/j.jadohealth.2008.10.002

31. Toh ZQ, Licciardi PV, Fong J, et al. Reduced dose human papillomavirus vaccination: an update of the current state-of-the-art. Vaccine. 2015;33(39):5042-5050. doi:10.1016/j.vaccine.2015.07.102

32. World Health Organization. Meeting of the Strategic Advisory Group Of Experts on immunization, April 2014 - conclusions and recommendations. Relevé Épidémiologique Hebdomadaire. 2014;89(21):221-236.

33. Brown DR, Kjaer SK, Sigurdsson K, et al. The impact of quadrivalent human papillomavirus (HPV; types 6, 11, 16, and 18) L1 virus-like particle vaccine on infection and disease due to oncogenic nonvaccine HPV types in generally HPV-naive women aged 16-26 years. J Infect Dis. 2009;199(7):926-935. doi:10.1086/597307

34. Wheeler CM, Kjaer SK, Sigurdsson K, et al. The impact of quadrivalent human papillomavirus (HPV; types 6, 11, 16, and 18) L1 virus-like particle vaccine on infection and disease due to oncogenic nonvaccine HPV types in sexually active women aged 16-26 years. J Infect Dis. 2009;199(7):936-944. doi:10.1086/597309

35. Paavonen J, Naud P, Salmeron J, et al. Efficacy of human papillomavirus (HPV)-16/18 AS04-adjuvanted vaccine against cervical infection and precancer caused by oncogenic HPV types (PATRICIA): final analysis of a double-blind, randomised study in young women. Lancet. 2009;374(9686):301-314. doi:10.1016/ S0140-6736(09)61248-4

36. Kavanagh K, Pollock KG, Cuschieri K, et al. Changes in the prevalence of human papillomavirus following a national bivalent human papillomavirus vaccination programme in Scotland: a 7-year cross-sectional study. Lancet Infect Dis. 2017;17(12):1293-1302. doi:10.1016/S1473-3099(17)30468-1

37. Barzon L, Squarzon L, Masiero S, et al. Neutralizing and cross-neutralizing antibody titres induced by bivalent and quadrivalent human papillomavirus vaccines in the target population of organized vaccination programmes. Vaccine. 2014;32(41):5357-5362. doi:10.1016/j.vaccine.2014.07.014

38. Garland SM, Cornall AM, Brotherton JML, et al. Final analysis of a study assessing genital human papillomavirus genoprevalence in young Australian women, following eight years of a national vaccination program. Vaccine. 2018;36(23):3221-3230. doi:10.1016/j. vaccine. 2018.04.080

39. Einstein $\mathrm{MH}$, Baron M, Levin MJ, et al. Comparison of the immunogenicity of the human papillomavirus (HPV)-16/18 vaccine and the HPV-6/11/16/18 vaccine for oncogenic non-vaccine types HPV-31 and HPV-45 in healthy women aged 18-45 years. Hum Vaccin. 2011;7(12):1359-1373. doi:10.4161/hv.7.12.18282

40. Godi A, Bissett SL, Miller E, Beddows S. Relationship between humoral immune responses against HPV16, HPV18, HPV31 and HPV45 in 12-15 year old girls receiving Cervarix(R) or Gardasil(R) vaccine. PLoS One. 2015;10(10):e0140926. doi:10.1371/journal.pone.0140926

41. Kemp TJ, Hildesheim A, Safaeian M, et al. HPV16/18 L1 VLP vaccine induces cross-neutralizing antibodies that may mediate cross-protection. Vaccine. 2011;29(11):2011-2014. doi:10.1016/j. vaccine.2011.01.001
42. Joura EA, Giuliano AR, Iversen OE, et al. A 9-valent HPV vaccine against infection and intraepithelial neoplasia in women. $N$ Engl J Med. 2015;372(8):711-723. doi:10.1056/NEJMoa1405044

43. Huh WK, Joura EA, Giuliano AR, et al. Final efficacy, immunogenicity, and safety analyses of a nine-valent human papillomavirus vaccine in women aged 16-26 years: a randomised, double-blind trial. Lancet. 2017;390(10108):2143-2159. doi:10.1016/S0140-6736(17)31821-4

44. Garland SM, Pitisuttithum P, Ngan HYS, et al. Efficacy, immunogenicity, and safety of a 9-valent human papillomavirus vaccine: subgroup analysis of participants from Asian countries. $J$ Infect Dis. 2018;218(1):95-108. doi:10.1093/infdis/jiy133

45. Ruiz-Sternberg AM, Moreira ED Jr., Restrepo JA, et al. Efficacy, immunogenicity, and safety of a 9-valent human papillomavirus vaccine in Latin American girls, boys, and young women. Papillomavirus Res. 2018;5:63-74. doi:10.1016/j.pvr.2017.12.004

46. Iwata S, Murata S, Rong Han S, Wakana A, Sawata M, Tanaka Y. Safety and Immunogenicity of a 9-valent human papillomavirus vaccine administered to 9- to 15-year-old Japanese girls. Jpn J Infect Dis. 2017;70 (4):368-373. doi:10.7883/yoken.JJID.2016.299

47. Van Damme P, Meijer C, Kieninger D, et al. A phase III clinical study to compare the immunogenicity and safety of the 9-valent and quadrivalent HPV vaccines in men. Vaccine. 2016;34 (35):4205-4212. doi:10.1016/j.vaccine.2016.06.056

48. Vesikari T, Brodszki N, van Damme P, et al. A randomized, double-blind, phase III study of the immunogenicity and safety of a 9-valent human papillomavirus L1 virus-like particle vaccine (V503) versus gardasil(R) in 9-15-year-old girls. Pediatr Infect Dis J. 2015;34(9):992-998. doi:10.1097/INF.0000000000000773

49. Luxembourg A, Ed M Jr., Samakoses R, et al. Phase III, randomized controlled trial in girls 9-15 years old to evaluate lot consistency of a novel nine-valent human papillomavirus L1 virus-like particle vaccine. Hum Vaccin Immunother. 2015;11(6):1306-1312. doi:10.1080/ 21645515.2015.1009819

50. Van Damme P, Olsson SE, Block S, et al. Immunogenicity and safety of a 9-valent HPV vaccine. Pediatrics. 2015;136(1):e28-39. doi:10.1542/peds.2014-3745

51. Luxembourg A, Brown D, Bouchard C, et al. Phase II studies to select the formulation of a multivalent HPV L1 virus-like particle (VLP) vaccine. Hum Vaccin Immunother. 2015;11(6):1313-1322. doi:10.1080/21645515.2015.1012010

52. Guevara A, Cabello R, Woelber L, et al. Antibody persistence and evidence of immune memory at 5years following administration of the 9-valent HPV vaccine. Vaccine. 2017;35(37):5050-5057. doi:10.1016/j.vaccine.2017.07.017

53. Castellsague X, Giuliano AR, Goldstone S, et al. Immunogenicity and safety of the 9-valent HPV vaccine in men. Vaccine. 2015;33 (48):6892-6901. doi:10.1016/j.vaccine.2015.06.088

54. Garland SM, Cheung TH, McNeill S, et al. Safety and immunogenicity of a 9-valent HPV vaccine in females 12-26 years of age who previously received the quadrivalent HPV vaccine. Vaccine. 2015;33 (48):6855-6864. doi:10.1016/j.vaccine.2015.08.059

55. Vatti A, Monsalve DM, Pacheco Y, Chang C, Anaya JM, Gershwin ME. Original antigenic sin: a comprehensive review. J Autoimmun. 2017;83:12-21. doi:10.1016/j.jaut.2017.04.008

56. Van Damme P, Bonanni P, Bosch FX, et al. Use of the nonavalent HPV vaccine in individuals previously fully or partially vaccinated with bivalent or quadrivalent HPV vaccines. Vaccine. 2016;34 (6):757-761. doi:10.1016/j.vaccine.2015.12.063

57. Iversen $\mathrm{OE}$, Miranda MJ, Ulied A, et al. Immunogenicity of the 9-valent HPV vaccine using 2-dose regimens in girls and boys vs a 3-dose regimen in women. JAMA. 2016;316(22):2411-2421. doi:10.1001/jama.2016.17615

58. Meites E, Kempe A, Markowitz L. se of a 2-dose schedule for Human Papillomavirus Vaccination - updated Recommendations of the Advisory Committee on Immunization Practices. MMWR Morb Mortal Wkly Rep. 2016;65:1405-1408. doi:10.15585/mmwr.mm6549a5 
59. European Medicines Agency Gardasil 9. Summary of product characteristics; 2018. Accessed October 29, 2018.

60. National Centre For Immunisation Research and Surveillance. Human papillomavirus (HPV) vaccines for Australians: information for immunisation providers; 2018. Accessed October 29, 2018.

61. Moreira ED Jr., Block SL, Ferris D, et al. Safety profile of the 9-Valent HPV vaccine: a combined analysis of 7 phase iii clinical trials. Pediatrics. 2016;138(2):e20154387. doi:10.1542/peds.2015-4387

62. Kosalaraksa P, Mehlsen J, Vesikari T, et al. An open-label, randomized study of a 9-valent human papillomavirus vaccine given concomitantly with diphtheria, tetanus, pertussis and poliomyelitis vaccines to healthy adolescents $11-15$ years of age. Pediatr Infect Dis J. 2015;34(6):627-634. doi:10.1097/INF.0000000000000694

63. Schilling A, Parra MM, Gutierrez M, et al. Coadministration of a 9-valent human papillomavirus vaccine with meningococcal and Tdap vaccines. Pediatrics. 2015;136(3):E563-E572. doi:10.1542/peds.2015-0573

64. Luxembourg A, Kjaer SK, Nygard M, et al. Design of a long-term follow-up effectiveness, immunogenicity and safety study of women who received the 9-valent human papillomavirus vaccine. Contemp Clin Trials. 2017;52:54-61. doi:10.1016/j.cct.2016.10.006

65. Markowitz LE, Gee J, Chesson H, Stokley S. Ten years of human papillomavirus vaccination in the United States. Acad Pediatr. 2018;18(2S):S3-S10. doi:10.1016/j.acap.2017.09.014

66. Ward K, Quinn H, Menzies R, McIntyre P. A history of adolescent school based vaccination in Australia. Commun Dis Intell $Q$ Rep. 2013;37(2):E168-E174.

67. McClure CA, MacSwain MA, Morrison H, Sanford CJ. Human papillomavirus vaccine uptake in boys and girls in a school-based vaccine delivery program in Prince Edward Island, Canada. Vaccine. 2015;33(15):1786-1790. doi:10.1016/j.vaccine.2015.02.047

68. Brotherton JM, Fridman M, May CL, Chappell G, Saville AM, Gertig DM. Early effect of the HPV vaccination programme on cervical abnormalities in Victoria, Australia: an ecological study. Lancet. 2011;377(9783):2085-2092. doi:10.1016/S0140-6736(11)60551-5

69. Machalek DA, Garland SM, Brotherton JML, et al. Very low prevalence of vaccine human papillomavirus (HPV) types among 18 to 35 year old Australian women, nine years following implementation of vaccination. $J$ Infect Dis. 2018. doi:10.1093/infdis/jiy075

70. Tabrizi SN, Brotherton JM, Kaldor JM, et al. Fall in human papillomavirus prevalence following a national vaccination program. $J$ Infect Dis. 2012;206(11):1645-1651. doi:10.1093/infdis/jis590

71. Patel C, Brotherton JM, Pillsbury A, et al. The impact of 10 years of human papillomavirus (HPV) vaccination in Australia: what additional disease burden will a nonavalent vaccine prevent? Euro Surveill. 2018;23:41. doi:10.2807/1560-7917.ES.2018.23.41.1700737

72. Brotherton JM, Hawkes D, Sultana F, et al. Age-specific HPV prevalence among 116,052 women in Australia's renewed cervical screening program: a new tool for monitoring vaccine impact. Vaccine. 2019;37(3):412-416. doi:10.1016/j.vaccine.2018.11.075

73. Hall MT, Simms KT, Lew JB, et al. The projected timeframe until cervical cancer elimination in Australia: a modelling study. Lancet Public Health. 2018;4(1):E19-E27. doi:10.1016/S2468-2667(18)30183-X
74. Gilca V, Sauvageau C, Trudeau G. Advisory report on the Human Papillomavirus (HPV) Vaccination Schedule. National Institute of Public Health of Quebec; 2018. Available from: https://www.inspq. qc.ca/sites/default/files/publications/2458_papillomavirus_vaccina tion_schedule.pdf

75. Gilca V, Sauvageau C, Panicker G, De Serres G, Ouakki M, Unger ER. Immunogenicity and safety of a mixed vaccination schedule with one dose of nonavalent and one dose of bivalent HPV vaccine versus two doses of nonavalent vaccine - A randomized clinical trial. Vaccine. 2018;36(46):7017-7024. doi:10.1016/j. vaccine.2018.09.057

76. Toh ZQ, Russell FM, Reyburn R, et al. Sustained antibody responses 6 years following 1, 2, or 3 doses of quadrivalent human papillomavirus (HPV) vaccine in adolescent Fijian girls, and subsequent responses to a single dose of bivalent HPV Vaccine: a prospective cohort study. Clin Infect Dis. 2017;64(7):852-859. doi:10.1093/cid/ciw865

77. Gilca V, Sauvageau C, Boulianne N, et al. The effect of a booster dose of quadrivalent or bivalent HPV vaccine when administered to girls previously vaccinated with two doses of quadrivalent HPV vaccine. Hum Vaccin Immunother. 2015;11(3):732-738. doi:10.1080/21645515.2015.1011570

78. Mennini FS, Bonanni P, Bianic F, et al. Cost-effectiveness analysis of the nine-valent HPV vaccine in Italy. Cost Eff Resour Alloc. 2017;15:11. doi:10.1186/s12962-017-0073-8

79. Largeron N, Petry KU, Jacob J, Bianic F, Anger D, Uhart M. An estimate of the public health impact and cost-effectiveness of universal vaccination with a 9-valent HPV vaccine in Germany. Expert Rev Pharmacoecon Outcomes Res. 2017;17(1):85-98. doi:10.1080/ 14737167.2016.1208087

80. Durham DP, Ndeffo-Mbah ML, Skrip LA, Jones FK, Bauch CT, Galvani AP. National- and state-level impact and cost-effectiveness of nonavalent HPV vaccination in the United States. Proc Natl Acad Sci U S A. 2016;113(18):5107-5112. doi:10.1073/pnas.1515528113

81. Petrosky E, Bocchini JA Jr., Hariri S, et al. Use of 9-valent human papillomavirus (HPV) vaccine: updated HPV vaccination recommendations of the advisory committee on immunization practices. MMWR Morb Mortal Wkly Rep. 2015;64(11):300-304.

82. Bruni L, Diaz M, Barrionuevo-Rosas L, et al. Global estimates of human papillomavirus vaccination coverage by region and income level: a pooled analysis. Lancet Glob Health. 2016;4(7):e453-e463. doi:10.1016/S2214-109X(16)30099-7

83. ClinicalTrials.gov. Scientific evaluation of one or two doses of the bivalent or nonavalent prophylactic HPV vaccines; 2018. Accessed October 7th, 2018.

84. ClinicalTrials.gov. A dose reduction immunobridging and safety study of two HPV vaccines in tanzanian girls (DoRIS); 2018. Accessed November 7th, 2018.

85. The World Health Organization. Vaccine in national immunization programme update; 2018. [Updated October 2018]. Accessed December 27th, 2018 .
Infection and Drug Resistance

\section{Publish your work in this journal}

Infection and Drug Resistance is an international, peer-reviewed openaccess journal that focuses on the optimal treatment of infection (bacterial, fungal and viral) and the development and institution of preventive strategies to minimize the development and spread of resistance. The journal is specifically concerned with the epidemiology of antibiotic resistance and the mechanisms of resistance development and diffusion in both hospitals and the community. The manuscript management system is completely online and includes a very quick and fair peerreview system, which is all easy to use. Visit http://www.dovepress.com/ testimonials.php to read real quotes from published authors. 